\title{
Inability to Digitize ECG Leads
}

National Cancer Institute

\section{Source}

National Cancer Institute. Inability to Digitize ECG Leads. NCI Thesaurus. Code $C 114170$.

A statement indicating the inability to digitize a paper ECG. (CDISC) 